\title{
Prevalence and Pattern of Peripheral Arterial Disease among Diabetic Individual in Al-jouf Region
}

\author{
Jarid Saleh Aljarid, Anwar Mohammed Alazmi, Ahmed Hammad Almaeen, Abdullah Odhayb \\ Alruwaili, Mostafa Mohamed Ragheb, Naif mousara Almousara \\ Department of Family and Community Medicine, Al-jouf University \\ Corresponding author: Jarid Saleh Aljarid, Email: j.aljared92@hotmail.com, Tel: +966508554501
}

\section{Abstract:}

Introduction: Peripheral arterial disease (PAD) is a common vascular disorder in Saudi Arabia where diabetes mellitus, hypertension and smoking are common risks. As this disease carries significant morbidity and mortality. Objective: This study aimed to screen for PAD in the community among diabetics, determining its prevalence, severity and associated risk factors. Patients and Methods: This study was done at 3 primary health care centers (PHCCs) at Domat Aljandal and two centers at Quraiat, Aljouf region. The included patients were 149, all were diabetic, 114 males and 35 females. The sociodemographic data, special habits, symptoms of peripheral ischemia and biochemical profiles [including blood lipids and fasting sugar] were extracted from the medical files and asked during interview. After clinical assessment, the ankle brachial index (ABI) was bilaterally determined on supine position using $8 \mathrm{MHz}$ vascular Doppler. PAD was diagnosed if an ABI was <0.9. Results: The means of age, duration of diabetes and body mass index were $59.2,9.13$ years and $30.07 \mathrm{~kg} / \mathrm{m}^{2}$ respectively. Of all, $47 \%$ were smokers and $56.7 \%$ had hypertension. Out of the 149 studied patients, $21(14.1 \%)$ were previously diagnosed to have PAD and had undergone angioplasty. Symptoms of PAD were found only in 10 of these 21 and none of the remaining 128. By the ABI measurement, PAD was diagnosed in 48 patients (32.2\%), being unilateral in 27 and bilateral in 21 patients. Most of PADs (79.2\%) were mild to moderate severity while it was severe $20.8 \%$. The frequency of PAD was nonsignificantly more among females, patients with co-morbid hypertension, patients on oral hypoglycemic drugs and smokers $(\mathrm{P}>0.05$ for each). The frequency of low $\mathrm{ABI}$ was significantly more frequent among patients with history of vascular surgery $(\mathrm{P}=0.001)$. Patients with $\mathrm{PAD}$ had significantly higher mean ages $(\mathrm{P}=0.048)$ and non- significantly higher mean of duration of $\mathrm{DM}$. If patients known to have PAD are excluded, new diagnosis of PAD was made in 30 of 128 naïve patients $(23.4 \%)$. Conclusion: The prevalence of PAD in diabetic individuals in the community is high, probably due to the high frequency of other risks such as smoking and hypertension. These finding warrants activation of the nationwide screening program for prevention of cardiovascular diseases to decrease the morbidity and mortality of this diabetic complication.

Keywords: Peripheral Arterial Disease, Diabetic Individual, Al-jouf.

\section{Introduction}

The peripheral arterial disease (PAD) is a pathological entity related toatherosclerotic and thromboembolic processes affecting the aorta, visceral branches and the arteries of the lower limbs ${ }^{(1-4) .}$ It is caused in most of cases by atherosclerosis, which leads to the development of narrowing and/ or occlusions in the major arteries of the lower limbs movement $(4,5)$. Significant narrowing leads to ischemia of tissues supplied by the affected artery, hence the signs and symptoms of the disease. The most common symptom of PAD is intermittent claudication (IC), whose prevalence is about $3 \%$ in patients with 40 years old that increases with age ${ }^{(3,4) \text {. }}$

Identification of risk factors is extremely important for the early diagnosis and treatment of PAD to slow reduced functional capacity and improve quality of life. Moreover, PAD has been related to an increase in cardiovascular morbidity and mortality, especially after acute myocardial infarction and/or stroke ${ }^{(4,6,7)}$. Basic clinical examination is usually based on symptoms such as IC, pain at rest and abnormal signs such as decreased peripheral pulses, ischemic ulceration and gangrene. PAD can be diagnosed in most cases by measuring the ankle-brachial index (3,6). Risk factors are cigarette smoking, diabetes mellitus, high blood pressure. About $3 \%$ of people under age 60 have PAD, and so do as many as $20 \%$ of those over age 70 . The disease is more common among males. However, later in life, higher proportions of women are affected (8) .Management of PAD is a two-step-process. First, reduction of the cardiovascular risk reduction through vascular risk factor modification and anti-platelet therapy and 
second, symptom-guided therapy including supervised exercise, pharmacological interventions and revascularization procedures, when needed ${ }^{(9)}$. Diabetes mellitus which is a common disease in KSA is a major risk factor for peripheral arterial disease through acceleration of the macrovascular changes ${ }^{(10)}$. Meanwhile due to change in the life style of Saudi citizens, hypertension is a common disease.

\section{Objective:}

This study aimed to determine the prevalence of PAD in the community among diabetic patients so as to detect early disease before it manifest clinically. The detailed objectives were to determine the prevalence of PAD among individuals with type 2 diabetes mellitus, to assess its severity and determine associated risk factors.

\section{Patients and Methods}

This community-based cross-sectional study was done in 5 primary health care centers, three at Domat Aljandal and two at Quraiat, Aljouf region. The study was approved by the committee of research and ethics of the college of medicine. Patients studied were 149, 35 females and 114 males, their age ranged 37 to 84 years and all had diabetes mellitus. Study procedures: After explaining the objectives and benefits of the study to eligible patients, the approved ones were interviewed regarding their sociodemographic data, special habits including smoking and symptoms suggestive of PAD. Their blood pressure was measured, and the ankle brachial index was measured during supine position using $8 \mathrm{MHz}$ vascular Doppler. The ankle-brachial index (ABI) is an efficient tool for objectively documenting the presence of lower-extremity peripheral arterial disease (PAD) ${ }^{(11,12)}$. It is a simple, reproducible, and cost-effective assessment that can be used to detect lower-extremity arterial stenosis in the primary care setting. An ABI less than 0.9 has been shown to have a sensitivity of $90 \%$ and a specificity of $98 \%$ for detecting a lower-extremity stenosis of greater than $50 \%$ $(13,14)$

\section{Statistics and Analysis:}

Statistics was done by using SPSS version 18 . Numeric variables were represented as mean $( \pm$ standard deviation), while non-numeric data was presented as frequency and percentage. Comparison between subgroups was done accordingly. A P value $<0.05$ was considered statistically significant. Results were presented as tables and graphs.

\section{Results:}

In this study, 149 patients were included 114 males and 35 females. Their age ranged 37-84 years with a mean of 59.2 years. All were diabetic and the duration of diabetes ranged between 1 to 31 years (mean $=9.13$ years). Of all patients, insulin was used to control blood sugar in $117(78.5 \%)$. Hypertension was present in 85 patients $(56.7 \%)$. Their body mass index ranged between 20.5 and $45.8 \mathrm{~kg} / \mathrm{m}^{2}$, with a mean of 30.07. Current smoking was present in $70(47 \%)$. Peripheral arterial disease was present in 48 patients $(32.2 \%)$, being bilateral in $21(14.1 \%)$ and unilateral in 27 (18.1\%). The arterial obstruction was mild to moderate in 39 of $48(81.2 \%)$ and severe in 9 $(18.8 \%)$. History of vascular surgery (angioplasty was found in 21 (14.1\%).

Peripheral arterial disease was more frequent among females 14/35 (40\%) than males 34/114 (29.8\%) with no statistically significant difference $(\mathrm{P}=0.303)$. Also, $\mathrm{PAD}$ was more frequent among diabetic patients with comorbid hypertension $37.6 \%$ (32/85) compared to $25(16 / 64)$ diabetics without hypertension $(\mathrm{P}>0.05)$, and more frequent patients on oral anti-diabetic drugs compared to insulin $(38.7 \%$ v.30.8\%, P>0.05). PAD was more frequent among non-insulin users compared to others (38.7 vs. $30.8 \%, \quad \mathrm{P}=0.389)$. PAD was significantly more frequent among patients with history of angioplasty compared to those without $(85.7 \%$ vs. $23.4 \%, \quad \mathrm{P}=0.001)$. Meanwhile, the prevalence of insulin users was significantly higher among females than males (94.3\% vs. $74.3 \%, P=0.009)$. The prevalence of PAD was more frequent in smokers than nonsmokers (37.1 vs. $27.8 \%$ ), with no statistically significant difference. In this study, the mean ages of patients with PAD was significantly higher compared to without PAD $(61.67 \pm 9.34$ vs. 58.05 $\pm 10.78, \mathrm{P}=0.048$ ). Meanwhile, the mean duration of diabetes mellitus was higher among PAD group compared to patients with no PAD (9.54 vs. 8.94), however, the difference showed no statistical significance. The mean values of $A B I$ were significantly higher in patients with PAD compared to patients with no PAD. The mean values of lipid profile and FBS showed no statistically significant difference between patients with and without PAD (Table $3)$. 
Table 1: Sociodemographic and clinical data of the studied population $(n=149)$

\begin{tabular}{|l|l|}
\hline Age range and mean (years) & $37-84(59 / 1 \pm 10.44)$ \\
\hline M:F & $114: 35$ \\
\hline Duration of DM range and mean (years) & $1-31(9.13 \pm 6.36)$ \\
\hline Insulin: oral therapy (N) & $117: 32$ \\
\hline Co-morbid hypertension (n \& \%) & $85(56.7 \%)$ \\
\hline BMI range and mean (Kg/m $\left.{ }^{2}\right)$ & $20.5-45.8$ \\
& $(30.26 \pm 6.62)$ \\
\hline Current smokers (n \& \%) & $70(47 \%)$ \\
\hline Past history of lower limb angioplasty(n \& \%) & $21(14.1 \%)$ \\
\hline PAD (n \& \%) & $48(32.2 \%)$ \\
\hline Unilateral: bilateral PAD (n) & $27: 21$ \\
\hline PAD mild to moderate: severe $(\mathrm{n})$ & $38: 10$ \\
\hline
\end{tabular}

Table 2: Relation of PAD with some variables among the studied population (n=149)

\begin{tabular}{|l|l|l|}
\hline & $\begin{array}{l}\text { PAD } \\
(\mathrm{n} \& \%)\end{array}$ & P value \\
\hline Males $(\mathrm{n}=114)$ & $34(29.8)$ & $>0.05$ \\
Females $(\mathrm{n}=35)$ & $14(40)$ & \\
\hline Diabetes $(\mathrm{n}=64)$ & $16(25)$ & $>0.05$ \\
Diabetes and Hypertension $(\mathrm{n}=84)$ & $32(37.6)$ & \\
\hline Insulin (n=117) & $36(30.8)$ & \\
oral hypoglycemic(n=31) & $31(38.7)$ & 0.05 \\
\hline History of vascular surgery (n=21) & $18(85.7)$ & \\
No history of vascular surgery (n=128) & $30(23.4)$ & $>0.05$ \\
\hline Smokers (n=70) & $26(37.1)$ & \\
Nonsmokers(n=79) & $22(27.8)$ & \\
\hline
\end{tabular}

Table 3: Mean differences in patients with PAD regarding age, BMI, duration of DM and Biochemical Profiles among the studied population $(n=149)$

\begin{tabular}{|c|c|c|c|c|}
\hline & & Mean & Std. Deviation & $P$ value \\
\hline \multirow{2}{*}{ Age } & PAD & 61.67 & 9.354 & \multirow[t]{2}{*}{0.048} \\
\hline & No PAD & 58.05 & 10.781 & \\
\hline \multirow{2}{*}{ Duration of DM } & PAD & 9.54 & 6.943 & \multirow[t]{2}{*}{0.592} \\
\hline & No PAD & 8.94 & 6.094 & \\
\hline \multirow{2}{*}{ SBP } & PAD & 150.27 & 24.181 & \multirow[t]{2}{*}{0.137} \\
\hline & No PAD & 144.23 & 22.53 & \\
\hline \multirow{2}{*}{ DBP } & PAD & 79.06 & 13.68 & \multirow[t]{2}{*}{0.682} \\
\hline & No PAD & 79.92 & 11.02 & \\
\hline \multirow{2}{*}{ BMI } & PAD & 29.29 & 5.63 & \multirow[t]{2}{*}{0.218} \\
\hline & No PAD & 30.72 & 7.02 & \\
\hline \multirow{2}{*}{$\mathrm{ABI} L t$} & PAD & 0.76 & 0.34 & \multirow[t]{2}{*}{0.001} \\
\hline & No PAD & 1.08 & 0.21 & \\
\hline \multirow{2}{*}{ ABI Rt } & PAD & 0.76 & 0.36 & \multirow[t]{2}{*}{0.001} \\
\hline & No PAD & 1.08 & 0.22 & \\
\hline \multirow{2}{*}{ Cholesterol } & PAD & 4.77 & 1.12 & \multirow[t]{2}{*}{0.108} \\
\hline & No PAD & 4.443 & 1.17 & \\
\hline \multirow{2}{*}{ FBS } & PAD & 186.73 & 68.05 & \multirow[t]{2}{*}{0.576} \\
\hline & No PAD & 180.17 & 66.25 & \\
\hline \multirow{2}{*}{ HDL } & PAD & 0.9 & 0.17 & \multirow[t]{2}{*}{0.185} \\
\hline & No PAD & 0.957 & 0.24 & \\
\hline
\end{tabular}

\section{Discussion:}

This study aimed to determine the prevalence and pattern of PAD among diabetic patients regularly attending the PHCCs at Aljouf region. The prevalence of PAD was high (32.2\%), being bilateral in 21 of 48 patients and severe in
10. PAD was significantly more frequent in patients with established diagnosis who previously had angioplasty. Other nonsignificant risks included female gender, comorbid hypertension, oral hypoglycemic drugs 
and smoking. The study also emphasized the importance of old age as an important risk factor. The results revealed by this work are an important warning message to health care providers and decision makers to the magnitude of PAD among diabetic patients at the level of PHCCs and community. Among asymptomatic 128 diabetic attendants, PAD was newly diagnosed in $23.4 \%$. The high prevalence of PAD could be explained by the inclusion of patients with type 2 diabetes mellitus who had high frequency of co-morbid hypertension and smoking. The prevalence of PAD in this study is higher than $11.7 \%$ of 471 attendants of PHCC as reported by Al-Sheikh and colleagues in $2007^{(15) .}$ In their study, DM was present in $50.1 \%$ and most of their patients were asymptomatic. In our study, only 10 of 21 previously diagnosed to have PAD and undergone angioplasty had symptoms while the remaining 128 patients showed no symptoms as revealed from their medical records or interview in the PHCCs. Among the 21 patients previously diagnosed as having PAD, low ABI was found in $18(85.7 \%)$. If such patients are excluded, the prevalence of PAD among undiagnosed cohort would be $23.4 \%$, all were asymptomatic. This prevalence is close to 23.1\% among 598 Saudi diabetic patients as reported by Alzahrani and colleagues in 2014 (16). However, the later study was hospitalbased. From many studies done in Saudi Arabia, the prevalence of PAD is increasing. If patients with PAD disease are not identified and managed, this cardiovascular complication of diabetes will represent a significant pressure on the health system in the near future. The absence of symptoms in our cohort of patients with PAD, could be either due to incomplete clinical assessment of diabetic patients or lack of recording data in their files. Patients with PAD whether manifest or non-manifest are at significant risk for increased morbidity and mortality due to complications of atherosclerosis as stroke and myocardial infarction, lower limb gangrene, ulceration and amputation ${ }^{(17,19)}$

Owing to the dramatic changes in lifestyle and diet in past decades, it is estimated that Saudi Arabia has 3.4 million cases of diabetes in 2015 (10). The Ministry of Health has implemented many national programs to for prevention of cardiovascular diseases and "The Crown's Health Prevention Program" for early detection and control of non-communicable diseases in the Kingdom. In view of our results, there is an urgent need to activate these efforts, increase awareness of health care providers to identify individuals with high PAD risk thus preventing onset of PAD particularly among patients with diabetes.

\section{Study limitations:}

Inclusion of 21 patients previously diagnosed with PAD, however, the prevalence of PAD was still high after their exclusion from the total sample.

The frequency of females was low due to social limitation and absence of female colleague in the research team.

In conclusion:

The prevalence of PAD is high in PHCCs at Aljouf region. This could be explained by the high frequency of co-morbid hypertension and smoking. The fact that most of our patients had no symptoms of PAD emphasized the importance of frequent screening for arterial patency particularly in diabetic.

\section{Recommendation:}

Improve recording of patients in their file. Physicians at the PHCCs should follow guidelines during their management of diabetics and high-risk patients and provide patients with health education to modify risk factors of PAD.

Provision of vascular Doppler to physicians in the PHCC as a sensitive, cheap, non-invasive tool for diagnosis of PAD.

\section{References}

1. Dieter RS, Chu WW, Pacanowski JP, McBride PE, and Tanke TE (2002): The significance of lower extremity peripheral arterial disease. Clin Cardiol., 25(1): 3-10.

2. Chobanian AV, Bakris GL, Black HR, Cushman WC, Green LA, et al (2003): The seventh report of the joint national committee on prevention, detection, evaluation, and treatment of high blood pressure: the JNC 7 report. JAMA., 289(19): 2560-2572.

3. Mahe G, Le Faucheur A, and NouryDesvaux B (2010): Ankle-brachial index and peripheral arterial disease. N Engl J Med., 2(5): 470-471.

4. Hirsch AT, Criqui MH, Treat-Jacobson D, Regensteiner JG, Creager MA et al. (2001): Peripheral arterial disease detection, awareness, and treatment in primary care. JAMA., 286(11): 1317-1324. 
5. McDermott MM, Greenland $P$, Liu $K$, Guralnik JM, Criqui MH, et al (2001): Leg symptoms in peripheral arterial disease: associated clinical characteristics and functional impairment. JAMA., 286(13): 15991606.

6. Fowkes FG, Housley E, Riemersma RA, Macintyre CC, Cawood EH et al. (1992): Smoking, lipids, glucose intolerance, and blood pressure as risk factors for peripheral atherosclerosis compared with ischemic heart disease in the Edinburgh Artery Study. Am J Epidemiol., 135(4): 331-340.

7. McDermott MM, Liu K, Criqui MH, Ruth K, Goff D et al. (2005): Ankle-brachial index and subclinical cardiac and carotid disease: the multi-ethnic study of atherosclerosis. Am J Epidemiol., 162(1): 33-41.

8. Sapoval MR1, Chatellier G, Long AL, Rovani C, Pagny JY, Raynaud AC, Beyssen BM, and Gaux JC (1996): "Self-expandable stents for the treatment of iliac artery obstructive lesions: long-term success and prognostic factors." AJR Am J Roentgenol., 166(5): 1173-1179.

9.https://www.jvascsurg.org/article/S07415214(06)02296-8/abstract?code=ymva-site 10. International Diabetic Federation (2016): Middle East and North Africa: http://www.idf.org/membership/mena/saudiarabia

11. Ankle-Brachial Index Measurement: http://emedicine.medscape.com/article/183944 9-overview\#a3

12. Lange SF, Trampisch HJ, Pittrow D et al. (2007): Profound influence of different methods for determination of the ankle brachial index on the prevalence estimate of peripheral arterial disease. BMC Public Health., https://bmcpublichealth.biomedcentral.com/art icles/10.1186/1471-2458-7-147

13. Shanmugasundaram M, Ram VK, Luft UC, Szerlip M and Alpert JS (2011): Peripheral arterial disease--what do we need to know?. Clin Cardiol., 34(8):478-82.

14. Yao ST, Hobbs JT and Irvine WT (1969): Ankle systolic pressure measurements in arterial disease affecting the lower extremities. Br J Surg., 56(9):676-9.

15. Al-Sheikh SO, Aljabri BA, Al-Ansary LA, Al-Khayal LA et al. (2007): Prevalence of and risk factors for peripheral arterial disease in Saudi Arabia A pilot cross-sectional study. Saudi Med J., 28 (3): 412-414

16. Alzahrani A1, Wang D, Bakhotmah B and Hu F (2014): Risk factors for peripheral artery disease among patients with diabetes in Saudi Arabia Hasan. Vascular Medicine, 19(2) 103-111.

17. The TASC Working Group (2011): Management of Peripheral Arterial Disease (PAD); Trans Atlantic Inter-Society Consensus (TASC). J Vasc Surg., 31: S1-S288.

18. Criqui MH, Langer RD, Fronek A, Feigelson HS, Klauber MR, McCann TJ et al .(1992): Mortality over a period of 10 years in patients with peripheral arterial disease. $\mathrm{N}$ Engl J Med., 326: 381-386.

19. Schaper N, Andros G, Apelqvist $\mathrm{J}$ et al .(2012): Diagnosis and treatment of peripheral arterial disease in diabetic patients with a foot ulcer. A progress report of the International Working Group on the Diabetic Foot. Diabetes Metab Res Rev., 28: 218-224. 